\title{
Skin Autofluorescence: A Pronounced Marker of Mortality in Hemodialysis Patients
}

\author{
Esther G. Gerrits ${ }^{a}$ b Helen L. Lutgers $^{a}$ Gertie H.W. Smeets ${ }^{c}$ \\ Klaas H. Groenier ${ }^{b}$ Andries J. Smit ${ }^{a}$ Reinold O.B. Gans ${ }^{a}$ \\ Henk J.G. Bilo ${ }^{\mathrm{a}-\mathrm{c}}$ \\ a Department of Internal Medicine, University Medical Center Groningen, Groningen, and \\ ${ }^{b}$ Diabetes Center and ${ }^{c}$ Department of Internal Medicine, Isala Clinics, Zwolle, The Netherlands
}

\section{Key Words}

Autofluorescence $\cdot$ Diabetes mellitus $\cdot$ Hemodialysis $\cdot$ Mortality

\begin{abstract}
Background: Accelerated formation and tissue accumulation of advanced glycation endproducts (AGEs), reflecting cumulative glycemic and oxidative stress, occur in age-related and chronic diseases like diabetes mellitus (DM) and renal failure, and contribute to vascular damage. Skin autofluorescence (AF), a noninvasive measurement method, reflects tissue accumulation of AGEs. The aim of our study was to determine the predictive value of skin AF on overall and cardiovascular mortality in hemodialysis patients. Methods: Baseline skin AF was measured in 105 patients on hemodialysis, 23 had DM. Survival status was assessed after a mean follow-up period of 4.9 years (interquartile range 2.3-6.9 years). Results: Multivariate Cox regression analysis showed skin AF (hazard ratio (HR) 1.83; 95\% confidence interval (CI) 1.32-2.54), preexisting cardiovascular disease (CVD) (HR 2.77; 95\% Cl 1.48-5.18), renal replacement therapy duration (HR $1.10 ; 95 \% \mathrm{Cl} 1.01-1.19)$, age (HR 1.03; 95\% Cl 1.01-1.06), serum albumin (HR 0.90; $95 \% \mathrm{Cl} 0.85-$ 0.95), hematocrit (HR 0.92; $95 \% \mathrm{Cl} 0.86-0.98)$, phosphorus (HR 2.01; $95 \% \mathrm{Cl} 1.15-3.49$ ), and parathyroid hormone (HR $0.99 ; 95 \% \mathrm{Cl} 0.98-0.996)$ to be predictors of mortality, whereas DM was not. Preexisting CVD and serum phosphorus were the only predictors of cardiovascular mortality. Conclusion: Skin AF showed to be an independent predictor of overall mortality in hemodialysis patients, but it had no predictive value for cardiovascular mortality.
\end{abstract}

Copyright $\odot 2012$ S. Karger AG, Basel 


\section{Introduction}

Overall and cardiovascular mortality rate is much higher in subjects with end-stage renal disease compared to the general population [1-4]. A contributing factor to the development of vascular damage is the formation and accumulation of advanced glycation endproducts (AGEs), which progressively occurs in all individuals with ageing. Accelerated formation and tissue accumulation of AGEs on proteins with slow turnover, occur in patients with chronic diseases like diabetes mellitus (DM), chronic renal failure, neurodegenerative diseases, and systemic inflammatory diseases. These AGEs are formed nonenzymatically under circumstances of glycemic or oxidative stress. Compared to healthy subjects, increased oxidative stress and reduced antioxidant levels have been found in patients with chronic kidney disease or those on hemodialysis. Oxidative stress, which accompanies uremia, increases the inflammatory state and promotes the alterations of certain molecules such as proteins, lipids, and carbohydrates [5-7]. Additionally, the impaired excretion of the breakdown products of AGE cross-linked proteins, the so-called AGE free peptides and adducts, further contributes to the accumulation of AGEs in patients with chronic kidney disease. AGEs formed under pro-inflammatory and pro-oxidative circumstances will contribute to endothelial dysfunction, and are associated with the occurrence of cardiovascular disease (CVD) $[8,9]$.

A substudy of the Diabetes Control and Complications Trial (DCCT) showed that AGE levels in skin biopsies predicted the risk of development or progression of microvascular disease in type $1 \mathrm{DM}$, an observation which is suggestive for the deleterious effects of AGEs on the vascular wall [10].

Tissue accumulation of AGEs can be assessed in skin biopsies, but this is an invasive method. The autofluorescence (AF) reader, a noninvasive device, is based on the fluorescence properties of certain AGEs. The degree of skin AF reflects the level of tissue accumulation of AGEs reasonably well. This method has been validated against specific AGE levels in skin biopsies in patients with diabetes, patients on hemodialysis and in healthy control subjects $[11,12]$. Cross-sectional and longitudinal analyses have already shown the relationship between skin AF as a marker of tissue AGEs, and vascular morbidity and mortality in $\mathrm{DM}$, and mortality in hemodialysis. Cross-sectional analyses also showed the association of skin AF with renal and cardiovascular risk factors in stage 3 and predialysis CKD patients [12-18].

The aim of the present study was to address the predictive value of skin AF on overall and cardiovascular mortality in hemodialysis patients.

\section{Subjects and Methods}

\section{Study Group}

Between August 2003 and February 2004, 105 hemodialysis patients who dialyzed in the hemodialysis center of the Isala Clinics in Zwolle, The Netherlands, were included in the study cohort. These hemodialysis patients had a three times weekly scheme with variable duration of dialysis, all were using biocompatible low-flux membranes. Patients with Fitzpatrick class V-VI skin type were excluded from participation, because of the reduced ability of the prototype AF reader to reliably measure AF in these dark skin types. This inability is due to the high absorption of both the excitation and emission light when using an ultraviolet light source with a certain peak intensity $[19,20]$.

At the end of follow-up, in January 2011, survival status of all patients was assessed. Approval by the local ethic committee had been obtained and informed consent was given by all of the included patients. 


\section{Data Collection and Definitions}

Clinical data and laboratory results were obtained at the time of the baseline skin AF measurement. Laboratory data included serum non-fasting total cholesterol, creatinine, urea, albumin, hemoglobin, hematocrit, calcium, phosphorus and parathyroid hormone (PTH), all measured according to the standard laboratory procedures. Physical assessment data included body mass index and blood pressure. Blood pressure assessment was the average of three measurements obtained after disconnection of the hemodialysis session in supine position, using an aneroid device, in the week before the skin AF measurement.

Diagnosis of DM was confirmed using ADA criteria. The European Dialysis and Transplant Association Codes were used to define the primary diagnosis of renal failure, subdivided in divisions: primary glomerulonephritis, interstitial nephropathies, multisystem diseases, DM and not known/other. Some DM patients needed dialysis due to another cause of renal disease than diabetic nephropathy.

CVD was defined as: ischemic heart disease (International Classification of diseases (ICD-9) codes 410-414 and/or a history of coronary artery bypass surgery or percutaneous coronary intervention), cerebrovascular accidents or peripheral vascular disease (clinical history of intermittent claudication, percutaneous transluminal angioplasty, bypass surgery and/or limb amputation). The presence of macrovascular complications was assigned when meeting at least one of the criteria for CVD.

\section{Skin Autofluorescence}

Skin AF was measured at the lower, non-fistula arm by the AF reader (prototype of the current AGE Reader; DiagnOptics BV, Groningen, The Netherlands), a noninvasive device which illuminates a skin surface of $\sim 1 \mathrm{~cm}^{2}$, with an excitation ultraviolet light source with peak intensity at $\sim 370 \mathrm{~nm}$. Emission light and reflected excitation light from the skin are measured with a spectrometer in the $300-600 \mathrm{~nm}$ range. AF was computed by dividing the average light intensity of the emission spectrum $(420-600 \mathrm{~nm})$ by the average light intensity of the excitation spectrum (300-420 nm), multiplied by hundred and expressed in arbitrary units (AU). Assessing skin AF is not a time-consuming method and the operation of the device requires no special training or skills. Reproducibility has been tested before and the interindividual measurements and intraindividual seasonal variance showed a mean relative error of $\sim 5 \%[21]$.

\section{Statistical Analyses}

Student's t test was used to compare groups with respect to quantitative variables, and Cox regression analysis was used to estimate the effect and the $95 \%$ confidence interval (CI) of each predictor: skin AF, serum albumin, DM, preexisting CVD, renal replacement therapy duration at baseline, age, pulse pressure, hematocrit, serum phosphorus and PTH on overall and cardiovascular mortality, both in a univariate and in a multivariate model correcting for all other predictors.

\section{Results}

Baseline characteristics are shown in table 1; patients were subdivided in a surviving patient group and a nonsurviving patient group. Mean age of all the 105 included patients was 65 years, $68 \%$ were male, and $93 \%$ were Caucasian ( $7 \%$ Asian). Causes of end-stage renal disease were classified in $9 \%$ of all patients as DM, in $31 \%$ as hypertension or renovascular disease, in $21 \%$ as primary glomerular disorders and in $39 \%$ as having another primary disorder causing end-stage renal disease. There were 23 type $2 \mathrm{DM}$ patients (there were no type $1 \mathrm{DM}$ 
Table 1. Baseline characteristics of hemodialysis patients (total, surviving patients and nonsurviving patients)

\begin{tabular}{lccc}
\hline Characteristic & $\begin{array}{c}\text { Surviving } \\
\text { hemodialysis } \\
\text { patients }(\mathrm{n}=36)\end{array}$ & $\begin{array}{l}\text { Nonsurviving } \\
\text { hemodialysis } \\
\text { patients }(\mathrm{n}=69)\end{array}$ & $\begin{array}{c}\text { Total } \\
(\mathrm{n}=105)\end{array}$ \\
\hline Age, years & $54.5 \pm 15.1$ & $70.5 \pm 10.9^{\mathrm{a}}$ & $65.1 \pm 14.6$ \\
Gender (M/F), \% & $58 / 42$ & $73 / 27$ & $68 / 32$ \\
Smoking, \% & 8 & 17 & 14 \\
Body mass index & $25.5 \pm 4.5$ & $24.6 \pm 4.5$ & $24.9 \pm 4.5$ \\
Systolic blood pressure, mm Hg & $140 \pm 22$ & $149 \pm 25$ & $146 \pm 24$ \\
Pulse pressure, mm Hg & $56 \pm 18$ & $70 \pm 20^{\mathrm{a}}$ & $65 \pm 20$ \\
Median duration of renal replacement & & & \\
$\quad$ therapy, years (interquartile range) & $2.94(0.61-5.46)$ & $2.56(1.21-5.72)$ & $2.64(1.11-5.54)$ \\
Preexisting CVD, \% & 17 & $67^{\mathrm{a}}$ & 50 \\
$\quad$ Coronary heart disease, \% & 8 & $45^{\mathrm{a}}$ & 32 \\
$\quad$ CVA/TIA, \% & 8 & 20 & 16 \\
$\quad$ Peripheral vascular disease, \% & 3 & $30^{\mathrm{a}}$ & 21 \\
Diabetes mellitus, \% & 8 & $29^{\mathrm{b}}$ & 22 \\
Creatinine, $\mu$ mol/l & $983 \pm 208$ & $816 \pm 232^{\mathrm{a}}$ & $873 \pm 237$ \\
Urea, mmol/l & $27.6 \pm 5.4$ & $26.0 \pm 6.9$ & $26.5 \pm 6.4$ \\
Albumin, g/l & $40 \pm 4$ & $37 \pm 5^{\mathrm{c}}$ & $38 \pm 5$ \\
Total cholesterol, mmol/l & $3.9 \pm 0.9$ & $3.8 \pm 1.0$ & $3.8 \pm 0.9$ \\
Hemoglobin, mmol/l & $7.9 \pm 0.7$ & $7.7 \pm 0.9$ & $7.8 \pm 0.8$ \\
Hematocrit, \% & $38.2 \pm 3.8$ & $37.8 \pm 4.6$ & $38.0 \pm 4.3$ \\
Calcium, mmol/l & $2.36 \pm 0.20$ & $2.34 \pm 0.20$ & $2.35 \pm 0.20$ \\
Phosphorus, mmol/l & $1.68 \pm 0.43$ & $1.63 \pm 0.58$ & $1.65 \pm 0.53$ \\
Median parathyroid hormone, pmol/l (range) & $19.7(4.7-60.8)$ & $15.6(6.9-33.6)$ & $16.9(6.6-39.5)$ \\
Skin AF, AU & $2.74 \pm 0.77$ & $3.37 \pm 0.86^{\mathrm{a}}$ & $3.16 \pm 0.88$ \\
\hline & & & \\
\hline
\end{tabular}

Data are mean $\pm \mathrm{SD}$, unless otherwise indicated. CVA/TIA = Cerebrovascular accident/transient ischemic attack. ${ }^{\mathrm{a}} \mathrm{p} \leq 0.001 ;{ }^{\mathrm{b}} \mathrm{p}=0.02 ;{ }^{\mathrm{c}} \mathrm{p}=0.03$ (compared to the surviving hemodialysis patients).

patients in our hemodialysis group at that time point) who appeared well controlled with a mean $\mathrm{HbA}_{1 \mathrm{c}} \pm \mathrm{SD}$ of $6.8 \pm 1.2 \%(51 \pm 13 \mathrm{mmol} / \mathrm{mol})$. Obviously, at baseline, the nonsurviving patients were older, had a higher percentage of DM and preexisting CVD, had a higher pulse pressure, a lower albumin level and a higher skin AF level. After a median follow-up time of 4.9 years (interquartile range $2.3-6.9$ years), the overall mortality rate was $66 \%$, and cardiovascular mortality rate was $32 \%$. Crude mortality rate was higher amongst the DM patients: $87 \%(20 / 23)$ versus $60 \%(49 / 82)$ in the non-DM group $(\mathrm{p}=0.02)$.

Univariate Cox regression analysis showed that preexisting CVD and skin AF were the most predictive markers of overall mortality: HR 3.44 (95\% CI 2.07-5.74), and 1.72 (95\% CI 1.32-2.23). DM status had statistically significant predictive value for overall mortality in univariate analysis, but not in multivariate Cox regression analysis (table 2), whereas skin $\mathrm{AF}$, serum albumin, preexisting $\mathrm{CVD}$, renal replacement therapy duration at baseline, age, hematocrit, serum phosphorus and PTH showed significant predictive value for overall mortality in the multivariate analysis. Surprisingly, median (interquartile range) PTH levels of the survivors were higher than those of the nonsurvivors: 19.7 (4.7-60.8) pmol/l versus 15.6 (6.9-33.6) $\mathrm{pmol} / \mathrm{l}$.

None of the variables showed significant predictive value for cardiovascular mortality in univariate Cox regression analysis. In multivariate analysis, only preexisting CVD and 
Table 2. Predictors of overall mortality by univariate and multivariate Cox regression analysis

\begin{tabular}{llcllc}
\hline Variable & Univariate & & & Multivariate & \\
\cline { 2 - 3 } & Hazard ratio (95\% CI) & $\mathrm{p}$ & & Hazard ratio (95\% CI) & $\mathrm{p}$ \\
\hline Skin AF, AU & $1.72(1.32-2.23)$ & $<0.001$ & & $1.83(1.32-2.54)$ & $<0.001$ \\
Diabetes mellitus (yes vs. no) & $1.93(1.15-3.26)$ & 0.01 & & $1.07(0.61-1.87)$ & 0.8 \\
Preexisting CVD (yes vs. no) & $3.44(2.07-5.74)$ & $<0.001$ & & $2.77(1.48-5.18)$ & 0.001 \\
RRT duration at baseline, years & $1.01(0.95-1.08)$ & 0.7 & & $1.098(1.01-1.19)$ & 0.02 \\
Age, years & $1.05(1.03-1.07)$ & $<0.001$ & & $1.03(1.01-1.06)$ & 0.02 \\
Pulse pressure, mm Hg & $1.02(1.01-1.03)$ & $<0.001$ & & $1.01(0.996-1.02)$ & 0.2 \\
Albumin, g/l & $0.93(0.89-0.97)$ & 0.001 & & $0.90(0.85-0.95)$ & $<0.001$ \\
Hematocrit, \% & $0.97(0.92-1.03)$ & 0.4 & & $0.92(0.86-0.98)$ & 0.008 \\
Serum phosphorus, mmol/1 & $0.85(0.51-1.40)$ & 0.5 & & $2.01(1.15-3.49)$ & 0.01 \\
Parathyroid hormone, pmol/1 & $0.99(0.99-1.00)$ & 0.07 & & $0.99(0.98-0.996)$ & 0.003 \\
\hline
\end{tabular}

RRT $=$ Renal replacement therapy.

serum phosphorus showed significant predictive value for cardiovascular mortality: HR 5.38 (95\% CI 1.31-22.19) and HR 3.59 (95\% CI 1.32-9.74).

During follow-up, 28 patients received a kidney transplant of whom 4 patients $(1 \mathrm{DM}, 3$ non-DM) had died at the end of follow-up. Performing Cox regression analyses in the population after exclusion of the transplanted hemodialysis patients did not change the initially identified predictive markers of overall mortality.

\section{Discussion}

This study showed that skin AF was significantly higher in the nonsurvivor group compared to the survivor group. Baseline skin AF was a predictor of overall mortality and showed independent predictive value regarding overall mortality besides the well-known risk factors serum albumin, preexisting CVD, renal replacement therapy duration at baseline, age, hematocrit, and serum phosphorus, with only little change in hazard ratio compared to the univariate analysis. DM per se, usually an important predictor of mortality in hemodialysis, did not independently have predictive power regarding mortality in the multivariate analysis, probably due to the small group size or to the more pronounced role of the other conventional and nonconventional cardiovascular risk factors in hemodialysis patients in general. We confirmed the independent predictive value of skin AF for overall mortality in hemodialysis patients as previous reported, but now with a slightly longer follow-up [12]. An explanation for the predictive value of skin AF, or AGE fluorescence, could be the intermediate role of AGEs in the development of vascular complications in DM, renal failure and CVD. Besides decreased clearance of AGEs in patients with renal failure, AGE formation is accelerated throughout the years during dialysis, resulting in progressive AGEs cross-linked to long-lived proteins embedded in tissue which may contribute to endothelial dysfunction. Additionally, high oxidative stress levels in patients with end-stage renal disease prior to the start of renal replacement therapy could contribute to increased AGE levels as well [6, 9, 22]. Recent data showed that high skin AF levels, as reflecting AGE accumulation, were found in moderate renal failure as well and were associated with renal and cardiovascular risk factors [17]. 
In contrast to our findings, previous studies on serum AGEs did not show predictive value concerning (cardiovascular) mortality or morbidity in chronic kidney disease [23, 24]. This might be due to AGE accumulation in skin collagen and other long-lived proteins which are supposed to remain more stable than serum AGE levels due to a variable clearance of circulating serum AGEs in patients with chronic kidney disease. Circulating serum AGEs apparently do not properly mirror chronic tissue AGE accumulation and its ensuing vascular damage on the long term. A recent report observed a relationship between diastolic dysfunction and skin AF in hemodialysis patients, whereas serum AGEs did not support this concept [25]. Another explanation for the higher variability of circulating serum AGEs in hemodialysis patients may be the use of different dialysis modalities [26-28].

Both PTH and phosphorus are well-known risk factors for mortality in hemodialysis patients. Although the two variables are most probably depending on each other, they both have their independent effect on mortality. PTH was negatively associated with mortality, with a wide spread of PTH levels in our study group, and with surprisingly higher levels of PTH in the survivor group. This paradoxical finding could be explained by the fact that part of the patients underwent a parathyroidectomy because of tertiary hyperparathyroidism, and lost their ability to produce large amounts of PTH, thus masking a possible correlation between severe parathyroid dysfunction and increased mortality risk.

Several factors may play an important role in the pathogenesis of the so-called 'chronic kidney disease'-associated wasting. Hypoalbuminemia is just one aspect occurring in correlation with those factors and therefore in line with our finding of being associated with mortality. Other factors contributing to protein-energy wasting in chronic kidney disease include systemic inflammation, changes in appetite-controlling hormones from reduced renal clearance, aberrant neuropeptide signalling, insulin and insulin-like growth factor resistance, and metabolic acidosis $[29,30]$. Consequently, hemodialysis patients are exposed to chronic inflammation, which might contribute to or overlap with the deleterious effects of oxidative stress in the development of arterial vascular disease. Unfortunately, we did not have sufficient data regarding nonconventional cardiovascular markers of oxidative stress or inflammation (like hs-CRP and IL-6).

There are some technical restrictions of the skin AF measurement. The skin AF reader may also measure fluorophores other than fluorescent AGEs, and there are nonfluorescent AGEs present in the skin as well that may contribute to the overall effects of AGE accumulation. Nevertheless, the predictive value of skin AF is repeatedly confirmed in several studies [12-16]. Moreover, only patients with skin type Fitzpatrick class I-IV could be included in our study at that time, because of the limitation of the prototype AF reader to measure in dark skin types. This issue was only just solved according to a recent report that skin AF can be measured in dark skin types with a newly developed AF reader [31]. Other limitations of the study are the small sample size and the possible selection bias of the hemodialysis patients with DM. Skin AF measurements were performed during hemodialysis, which theoretically could temporarily increase skin AF levels in association with higher oxidative stress levels induced by dialysis. However, no changes in skin AF before and after hemodialysis sessions could be observed, both in high- and low-flux dialysis settings (unpublished data: Koetsier M: Optical skin spectra reflect changes in tissue after hemodialysis).

In our opinion, our study provides further support for the concept of chronic inflammation and oxidative stress, both contributors to accelerated AGE formation and accumulation, as important nonconventional cardiovascular risk markers of mortality in a hemodialysis population. Measuring skin AF is an elegant and noninvasive method for the assessment of the amount of tissue AGEs, contributing to identify the mortality risk in hemodialysis patients. 
To summarize, risk factors which activate chronic inflammation and/or oxidative stress, contribute to the deleterious effects on the vascular wall in patients on hemodialysis. When correcting for other known risk factors, skin AF proved to be a pronounced marker of overall mortality in hemodialysis patients, whereas DM, another well-known risk factor, did not play a role in our study group.

\section{Financial Support}

This study was supported by grants of the Center of Excellence for Diabetes of the Isala Clinics, Zwolle, The Netherlands, and the Research Foundation of the Zwolle Internist Federation, Zwolle, The Netherlands.

\section{Disclosure Statement}

A.J. Smit is founder of DiagnOptics B.V., Groningen, The Netherlands, manufacturer of the AGE-Reader, which is based on the prototype used in the present article.

\section{References}

1 Foley RN, Parfrey PS, Sarnak MJ: Epidemiology of cardiovascular disease in chronic renal disease. J Am Soc Nephrol 1998;9:S16-S23.

-2 Goodkin DA, Young EW, Kurokawa K, Prütz KG, Levin NW: Mortality among hemodialysis patients in Europe, Japan, and the United States: case-mix effects. Am J Kidney Dis 2004;44:S16-S21.

-3 Go AS, Chertow GM, Fan D, McCulloch CE, Hsu CY: Chronic kidney disease and the risks of death, cardiovascular events and hospitalization. N Engl J Med 2004;351:1296-1305.

-4 Henry RM, Kostense PJ, Bos G, et al: Mild renal insufficiency is associated with increased cardiovascular mortality: The Hoorn Study. Kidney Int 2002;62:1402-1407.

-5 Toborek M, Wasik T, Drózdz M, Klin M, Magner-Wróbel K, Kopieczna-Grzebieniak E: Effect of hemodialysis on lipid peroxidation and antioxidant system in patients with renal failure. Metabolism 1992;41:1229-1232.

-6 Miyata T, Wada Y, Cai Z, et al: Implication of an increased oxidative stress in the formation of advanced glycation end products in patients with end-stage renal failure. Kidney Int 1997;51:1170-1181.

7 Coaccioli S, Standoli ML, Biondi R, et al: Assessment of the oxidative stress markers in patients with chronic renal insufficiency undergoing dialysis treatment. Clin Ter 2010;161:441-444.

-8 Zieman SJ, Kass DA: Advanced glycation endproduct crosslinking in the cardiovascular system: potential therapeutic target for cardiovascular disease. Drugs 2004;64:459-470.

-9 Linden E, Cai W, He JC, et al: Endothelial dysfunction in patients with chronic kidney disease results from advanced glycation end products (AGE)-mediated inhibition of endothelial nitric oxide synthase through RAGE activation. Clin J Am Soc Nephrol 2008;3:691-698.

-10 Genuth S, Sun S, Cleary P, et al; DCCT Skin Collagen Ancillary Study Group: Glycation and carboxymethyllysine levels in skin collagen predict the risk of future 10-year progression of diabetic retinopathy and nephropathy in the diabetes control and complications trial and epidemiology of diabetes interventions and compliations participants with type 1 diabetes. Diabetes 2005;54:3103-3111.

- 11 Meerwaldt R, Graaff R, Oomen PH, et al: Simple non-invasive assessment of advanced glycation endproduct accumulation. Diabetologia 2004;47:1324-1330.

-12 Meerwaldt R, Hartog JW, Graaff R, et al: Skin autofluorescence, a measure of cumulative metabolic stress and advanced glycation end products, predicts mortality in hemodialysis patients. J Am Soc Nephrol 2005;16:3687-3693.

13 Meerwaldt R, Lutgers HL, Links TP, et al: Skin autofluorescence is a strong predictor of cardiac mortality in diabetes. Diabetes Care 2007;30:107-112. 
-14 Gerrits EG, Lutgers HL, Kleefstra N, et al: Skin autofluorescence: a tool to identify type 2 diabetic patients at risk for developing microvascular complications. Diabetes Care 2008;31:517-521.

-15 Lutgers HL, Graaff R, Links TP, et al: Skin autofluorescence as a non-invasive marker of vascular damage in patients with type 2 diabetes mellitus. Diabetes Care 2006;29:2654-2659.

-16 Tanaka K, Katoh T, Asai J, et al: Relationship of skin autofluorescence to cardiovascular disease in Japanese hemodialysis patients. Ther Apher Dial 2010;14:334-340.

-17 McIntyre NJ, Fluck RJ, McIntyre CW, Taal MW: Skin autofluorescence and the association with renal and cardiovascular risk factors in chronic kidney disease stage 3. Clin J Am Soc Nephrol 2011;6: 2356-2363.

-18 Tanaka K, Tani Y, Asai J, et al: Skin autofluorescence is associated with renal function and cardiovascular diseases in pre-dialysis chronic kidney disease patients. Nephrol Dial Transplant 2011;26: $214-220$.

-19 Fitzpatrick TB: The validity and practicability of sun-reactive skin types I through VI. Arch Dermatol 1988;124:869-871.

-20 Kawada A: Risk and preventive factors for skin phototype. J Dematol Sci 2000;23:S27-S29.

-21 Meerwaldt R, Links T, Graaff R, et al: Simple noninvasive measurement of skin autofluorescence. Ann N Y Acad Sci 2005;1043:290-298.

-22 Diepeveen SH, Verhoeven GH, van der Palen J, et al: Oxidative stress in patients with end-stage renal disease prior to the start of renal replacement therapy. Nephron Clin Pract 2004;98:c3-c7.

-23 Schwedler SB, Metzger T, Schinzel R, Wanner C: Advanced glycation end products and mortality in hemodialysis patients. Kidney Int 2002;62:301-310.

- 24 Busch M, Franke S, Müller A, et al: Potential cardiovascular risk factors in chronic kidney disease: AGEs, total homocysteine and metabolites, and the C-reactive protein. Kidney Int 2004;66:338-347.

-25 Hartog JW, Hummel YM, Voors AA, et al: Skin-autofluorescence, a measure of tissue advanced glycation end-products (AGEs), is related to diastolic function in dialysis patients. J Card Fail 2008;14: 596-602.

-26 Kalousová M, Zima T, Tesar V, Lachmanová J: Advanced glycation end products and advanced oxidation protein products in hemodialyzed patients. Blood Purif 2002;20:531-536.

-27 Fagugli RM, Vanholder R, De Smet R, et al: Advanced glycation end products: specific fluorescence changes of pentosidine-like compounds during short daily hemodialysis. Int J Artif Organs 2001;24: 256-262.

28 Inagi R, Miyata T: Oxidative protein damage with carbohydrates and lipids in uremia: 'Carbonyl stress', Blood Purif 1999;17:95-98.

-29 Honda H, Qureshi AR, Heimbürger O, et al: Serum albumin, C-reactive protein, interleukin 6, and fetuin A as predictors of malnutrition, cardiovascular disease, and mortality in patients with ESRD. Am J Kidney Dis 2006;47:139-148.

-30 Mak RH, Ikizler AT, Kovesdy CP, Raj DS, Stenvinkel P, Kalantar-Zadeh K: Wasting in chronic kidney disease. J Cachexia Sarcopenia Muscle 2011;2:9-25.

-31 Koetsier M, Nur E, Chunmao H, et al: Skin color independent assessment of aging using skin autofluorescence. Opt Express 2010;18:14416-14429. 